\title{
ANOSOV FLOWS AND EXPANSIVENESS
}

\author{
VICTOR NORTON AND THOMAS O'BRIEN ${ }^{1}$
}

\begin{abstract}
We prove that an Anosov diffeomorphism of a compact manifold is expansive. We also show that a continuous flow on an infinite compact metric space cannot be expansive. We define a corresponding expansive concept for flows, that of an unstable flow (the word unstable is used here in a Lyapunov sense). We then prove that Anosov flows of compact manifolds are unstable.
\end{abstract}

1. Introduction. Let $X$ be a metric space with metric $d$ and $G$ a topological group. A transformation group $(X, G, \pi)$ is called expansive if there is an $\varepsilon>0$ (called an expansive constant) such that for any pair of distinct points $x, y$ in $X$ there is a $g \in G$ such that $d(x g, y g)>\varepsilon$. Here, as is customary, $x g$ is used in place of $\pi(x, g)$.

If $G$ is the integers then the transformation group is generated by a homeomorphism $f: X \rightarrow X$. In this case $f$ is called an expansive homeomorphism. There are numerous examples of expansive homeomorphisms on compact spaces [2]. On the other hand if $G$ is the reals $R$ and $X$ is an infinite compact space then $(X, R, \pi)$ cannot be expansive (Proposition 3.1). When $G=R$, the transformation group will be called a flow. For flows, we will use $\gamma(x)$ to denote the orbit of the point $x$.

Definition 1.1. A flow $\pi: X \times R \rightarrow X$ is called unstable if there is an $\varepsilon>0$ such that for any $x, y$ in $X$ with $y \notin \gamma(x)$ there is a $t$ (possibly negative) for which $d(x t, y t)>\varepsilon$. Thus, if $\pi$ is unstable, no orbit can be both positively and negatively Lyapunov stable (here we assume $X$ is self-dense; $X$ will be a manifold for the remainder of the paper). In $\$ 2$ we prove that an Anosov diffeomorphism on a compact manifold is expansive. This is indicated in [3, Proposition 8.7] but a proof has not been published. In $\S 3$ we prove that an Anosov flow on a compact manifold is unstable.

2. Anosov diffeomorphisms. A diffeomorphism $f$ of a compact Riemannian manifold $M$ is called an Anosov diffeomorphism if there is a splitting of the tangent bundle $T M=E^{u}+E^{s}$, invariant under the derivative $D f$,

Received by the editors January 18, 1973.

AMS (MOS) subject classifications (1970). Primary 58F15, 54H20; Secondary 57D50.

Key words and phrases. Anosov diffeomorphism, Anosov flow, expansive, unstable.

1 The second named author was supported by NSF Grant GP-28721.

(c) American Mathematical Society 1973 
and positive numbers $a, b$, and $\lambda$ such that:

$$
\begin{aligned}
& \left\|D f^{n} \cdot \xi^{u}\right\| \geqq a e^{\lambda n}\left\|\xi^{u}\right\|, \quad\left\|D f^{-n} \cdot \xi^{u}\right\| \leqq b e^{-\lambda n}\left\|\xi^{u}\right\|, \\
& \left\|D f^{n} \cdot \xi^{s}\right\| \leqq b e^{-\lambda n}\left\|\xi^{s}\right\|, \quad\left\|D f^{-n} \cdot \xi^{s}\right\| \geqq a e^{\lambda n}\left\|\xi^{s}\right\|,
\end{aligned}
$$

for every $\xi=\xi^{u}+\xi^{s}$ in $T M$.

We will assume that

$$
\begin{aligned}
\left\|D f \cdot \xi^{u}\right\| & \geqq 7\left\|\xi^{u}\right\|, \quad\left\|D f^{-1} \cdot \xi^{u}\right\| \leqq \frac{1}{7}\left\|\xi^{u}\right\|, \\
\left\|D f \cdot \xi^{s}\right\| & \leqq \frac{1}{7}\left\|\xi^{s}\right\|, \quad\left\|D f^{-1} \cdot \xi^{s}\right\| \geqq 7\left\|\xi^{s}\right\|,
\end{aligned}
$$

for each $\xi$ in $T M$. These inequalities hold for some iterate of $f$, and $f$ is expansive if that iterate of $f$ is expansive.

This last set of inequalities implies that, given any tangent vector $\xi$ in $T M,\|D f \cdot \xi\| \geqq 3\|\xi\|$ if $\left\|\xi^{u}\right\| \geqq\left\|\xi^{s}\right\|$ and $\left\|D f^{-1} \cdot \xi\right\| \geqq 3\|\xi\|$ if $\left\|\xi^{s}\right\| \geqq\left\|\xi^{u}\right\|$. For instance when $\left\|\xi^{u}\right\| \geqq\left\|\xi^{s}\right\|$ we have

$$
\begin{aligned}
\|D f \cdot \xi\| & =\left\|D f \cdot \xi^{u}+D f \cdot \xi^{s}\right\| \geqq\left\|D f \cdot \xi^{u}\right\|-\left\|D f \cdot \xi^{s}\right\| \\
& \geqq 7\left\|\xi^{u}\right\|-\frac{1}{7}\left\|\xi^{s}\right\| \geqq 6\left\|\xi^{u}\right\| \geqq 3\|\xi\| .
\end{aligned}
$$

Let us denote the zero cross-section of $T M$ by $0_{*}$. For $r>0$ and $p$ in $M, B(r)_{p}$ is the open ball of radius $r$ about 0 in $T M_{p}$. We let $B(r)$ denote the open tube of radius $r$ about $0_{*}$ in $T M, B(r)=\bigcup B(r)_{p}(p \in M)$.

Let $\pi: T M \rightarrow M$ be the projection and $\exp$ the exponential map. The map $\Psi: \xi \rightarrow(\pi \xi$, exp $\xi)$ restricts to a diffeomorphism of some open neighborhood of $0_{*}$ in $T M$ onto an open neighborhood of the diagonal in $M \times M$. In order to simplify later measurements we lift $f$ and $f^{-1}$ to $T M$ via $\Psi$, defining $F=\Psi^{-1} \cdot(f \times f) \cdot \Psi, G=\Psi^{-1} \cdot\left(f^{-1} \times f^{-1}\right) \cdot \Psi$. Then if $r$ is taken sufficiently small, $F$ and $G$ restrict to diffeomorphisms of $B(r)$ onto open neighborhoods $U$ and $V$ of $0_{*}$ in $T M$. We assume further that $d(\pi \xi$, $\exp \xi)=\|\xi\|$ for any $\xi$ in $B(r) \cup U \cup V$.

Let $F_{p}$ and $G_{p}$ denote the restrictions of $F$ and $G$ to the fibre $B(r)_{p}$. Then $F_{p}$ and $G_{p}$ are maps of Euclidean spaces. We have the relations:

$$
D F_{p}(0) \cdot \xi=D f \cdot \xi, \quad D G_{p}(0) \cdot \xi=D f^{-1} \cdot \xi
$$

for every $p$ in $M$ and $\xi$ in $T M_{p}$. These equations are a consequence of the chain rule and the fact that $D\left(\exp _{p}\right)(0): T M_{p} \rightarrow T M_{p}$ is the identity map.

Lemma 2.1. Let $f: M \rightarrow M$ be an Anosov diffeomorphism of the compact Riemannian manifold $M$. There is a positive number $\varepsilon$ such that either $d(f(p), f(q)) \geqq 2 d(p, q)$ or $d\left(f^{-1}(p), f^{-1}(q)\right) \geqq 2 d(p, q)$ whenever $d(p, q)<\varepsilon$.

PRoOF. The map $\xi \rightarrow\left\|D F_{\pi \xi}(\xi)-D F_{\pi \xi}(0)\right\|$ of $B(r)$ into $R$ is continuous and by compactness we can choose a number $\varepsilon_{F}$ less than or equal to $r$ such that $\left\|D F_{\pi \xi}(\xi)-D F_{\pi \xi}(0)\right\| \leqq 1$ for any $\xi$ in $B\left(\varepsilon_{F}\right)$ (the norm of the linear transformation $A=D F_{\pi \xi}(\xi)-D F_{\pi \xi}(0)$ of $T M_{\pi \xi}$ is the usual sup norm, $\|A\|=\sup \{\|A \cdot \eta\|:\|\eta\| \leqq 1\})$. Likewise choose $\varepsilon_{G}$ less than or equal 
to $r$ such that $\left\|D G_{\pi \xi}(\xi)-D G_{\pi \xi}(0)\right\| \leqq 1$ when $\xi$ is in $B\left(\varepsilon_{G}\right)$. Then setting $\varepsilon=\min \left\{\varepsilon_{F}, \varepsilon_{G}\right\}$, we have the estimates [1, I, $\S 4$, Corollary 2],

$$
\left\|F_{p}(\xi)-D f \cdot \xi\right\| \leqq\|\xi\|, \quad\left\|G_{p}(\xi)-D f^{-1} \cdot \xi\right\| \leqq\|\xi\|,
$$

for every $p$ in $M$ and $\xi$ in $B(\varepsilon)_{p}$.

Now suppose $p$ and $q$ are given with $d(p, q)<\varepsilon$. Take $\xi$ in $B(\varepsilon)_{p}$ satisfying $\exp \xi=q$. If $\left\|\xi^{\imath}\right\| \geqq\left\|\xi^{s}\right\|$,

$$
\begin{aligned}
d(f(p), f(q)) & =\left\|F_{p}(\xi)\right\| \geqq\|D f \cdot \xi\|-\|\xi\| \\
& \geqq 3\|\xi\|-\|\xi\|=2\|\xi\|=2 d(p, q) .
\end{aligned}
$$

On the other hand if $\left\|\xi^{s}\right\| \geqq\left\|\xi^{u}\right\|$ we have $d\left(f^{-1}(p), f^{-1}(q)\right) \geqq 2 d(p, q)$ by an analogous computation.

Applying Lemma 2.1 repeatedly we have:

Theorem 2.2. An Anosov diffeomorphism of a compact manifold $M$ is expansive (the $\varepsilon$ of Lemma 2.1 is an expansive constant).

3. Anosov flows. In this section $M$ is again a compact Riemannian manifold and the notations are the same as in $\S 2$.

Proposition 3.1. A flow on an infinite compact space cannot be expansive.

Proof. Let $X$ be infinite and compact and $\phi: X \times R \rightarrow X$ a flow. Let $\varepsilon$ be greater than $0, I=[0,1]$, and $\psi=\left.\phi\right|_{X \times I}$. Then if $\rho$ is the product metric on $X \times I$, there is a $\delta>0$ such that $\rho\left(\left(y, t_{1}\right),\left(z, t_{2}\right)\right)<\delta$ implies $d\left(y t_{1}, z t_{2}\right)<\varepsilon$. If $\phi$ is to be expansive there can be only finitely many rest points. Let $x$ be a nonrest point and choose a sequence of positive numbers $\left\{t_{n}\right\}$ decreasing monotonically to 0 so that $x \neq x t_{n}$ for any $n$. If $\varepsilon$ is an expansive constant for each $n$ there is an $s_{n}$ such that $d\left(x s_{n},\left(x t_{n}\right) s_{n}\right)>\varepsilon$. However for $n$ large enough $\rho\left(\left(x s_{n}, 0\right),\left(x s_{n}, t_{n}\right)\right)<\delta$ and hence $d\left(x s_{n},\left(x s_{n}\right) t_{n}\right)=$ $d\left(x s_{n},\left(x t_{n}\right) s_{n}\right)<\varepsilon$.

A differentiable flow $\phi: M \times R \rightarrow M$ induced by a vector field $\mathscr{V}$ is an Anosov flow if

(i) $\phi$ has no rest points,

(ii) there is an invariant decomposition $T M=E^{s}+E^{u}+E^{0}$ satisfying the following: there exist positive constants $a, b$, and $\lambda$ such that for any $\xi=\xi^{s}+\xi^{u}+\xi^{0}$ in $T M$,

$$
\begin{aligned}
& \left\|D \phi_{t} \cdot \xi^{u}\right\| \geqq a e^{\lambda t}\left\|\xi^{u}\right\| \quad \text { for all } t \geqq 0 \text {, } \\
& \left\|D \phi_{t} \cdot \xi^{s}\right\| \leqq b e^{-\lambda t}\left\|\xi^{s}\right\| \quad \text { for all } t \geqq 0 \text {, } \\
& \left\|D \phi_{t} \cdot \xi^{u}\right\| \leqq b e^{-\lambda t}\left\|\xi^{u}\right\| \quad \text { for all } t \leqq 0 \text {, } \\
& \left\|D \phi_{t} \cdot \xi^{s}\right\| \geqq a e^{\lambda t}\left\|\xi^{s}\right\| \quad \text { for all } t \leqq 0 \text {. }
\end{aligned}
$$


(iii) $E^{0}$ has dimension one and is tangent to the flow.

Note we can always choose a positive number $T$ so that

$$
\begin{aligned}
\left\|D \phi_{T} \cdot \xi^{u}\right\| & >7\left\|\xi^{u}\right\|, \\
\left\|D \phi_{T} \cdot \xi^{s}\right\|<\frac{1}{7}\left\|\xi^{s}\right\|, & \left\|D \phi_{-T} \cdot \xi^{u}\right\|<\frac{1}{7}\left\|\xi^{u}\right\|,
\end{aligned}
$$

There is a local codimension one submanifold $N_{p}$ at $p$ whose tangent space at $p$ is $E_{p}^{s}+E_{p}^{u} . N_{p}$ is transverse to the trajectories of the flow and is the image under $\exp _{p}$ of a neighborhood of 0 in $E_{p}^{s}+E_{p}^{u}$.

Lemma 3.3. Let $\phi: M \times R \rightarrow M$ be an Anosov flow. There exist $T$ and $\varepsilon$ greater than 0 such that if $p$ is in $M$ and $q$ is in $N_{p}$ and $d(p, q)<\varepsilon$ then either $d\left(\phi_{T}(p), \phi_{T}(q)\right) \geqq 2 d(p, q)$ or $d\left(\phi_{-T}(p), \phi_{-T}(q)\right) \geqq 2 d(p, q)$.

Proof. Let $T$ be chosen so that the inequalities, 3.2, are satisfied. Then the proof is essentially the same as that of Lemma 2.1 with $\phi_{T}$ replacing $f$ and $\phi_{-T}$ replacing $f^{-1}$. In particular, the $\varepsilon$ of this lemma, and of the following theorem, is small enough so that the exponential map is defined on the $\varepsilon$-tube about the zero section of the tangent bundle of $M$.

THEOREM 3.4. An Anosov flow on a compact manifold $M$ is unstable.

Proof. Let $\phi: M \times R \rightarrow M$ be an Anosov flow and let $\varepsilon$ and $T$ be as in the preceding lemma. Choose $\alpha>0$ such that if $d(p, q)<\alpha$ then $d\left(\phi_{T}(p), \phi_{T}(q)\right)<\varepsilon / 2$ and $d\left(\phi_{-T}(p), \phi_{-T}(q)\right)<\varepsilon / 2$. Now choose $\eta>0$ such that if $d(p, q)<\eta$ then there is a $q_{p}$ in $\gamma(q)$ with $q_{p}$ in $N_{p}$ and $d\left(q, q_{p}\right)<\alpha$. The existence of $\eta$ is guaranteed by the fact that trajectories are transverse to $N_{p}$. Let $\delta=\min (\eta, \varepsilon / 2)$. Suppose $p, q$ are in $M, q \notin \gamma(p)$ and $d(p, q)<\delta$ By the lemma either $d\left(\phi_{T}(p), \phi_{T}\left(q_{p}\right)\right)$ or $d\left(\phi_{-T}(p), \phi_{-T}\left(q_{p}\right)\right)$ is greater than $2 d\left(p, q_{p}\right)$. Also $d\left(\phi_{ \pm T}(q), \phi_{ \pm T}\left(q_{p}\right)\right)<\varepsilon / 2$. Thus for some integer $k$ we must have either $d\left(\phi_{k T}(p), \phi_{k T}(q)\right)>\eta$ and we cannot guarantee the existence of $q_{p}$ or else $d\left(\phi_{k T}(p), \phi_{k T}(q)\right)>\varepsilon / 2$ and the exponential map is no longer defined. Thus $\delta$ is an instability constant for $\phi$ and the proof is completed.

\section{REFERENCES}

1. S. Lang, Introduction to differentiable manifolds, Interscience, New York, 1962. MR 27 \#5192.

2. T. O'Brien and W. Reddy, Each compact orientable surface of positive genus admits an expansive homeomorphism, Pacific J. Math. 35 (1970), 737-741. MR 43 \#2692.

3. S. Smale, Differentiable dynamical systems, Bull. Amer. Math. Soc. 73 (1967), 747-817. MR 37 \#3598.

Department of Mathematics, Bowling Green State University, Bowling Green, Оhio 43403 